\title{
Effects of mindfulness-based stress reduction for adults with sleep disturbance: a protocol for an update of a systematic review and meta-analysis
}

Seong Min Kim ${ }^{1,2+}$, Jeong Min Park ${ }^{1+}$ and Hyun-Ju Seo ${ }^{3^{*}}$ (D)

\begin{abstract}
Background: Sleep disturbance is a common and significant health problem that has been linked to decreased quality of life. Mindfulness-based stress reduction (MBSR) can be a potentially effective intervention for insomnia. In previous systematic review examining the effects of MBSR for people with sleep disturbance, the authors highlighted the need for additional well-designed randomized controlled trials (RCTs) to assess the effects of MBSR practice. Recently, several RCTs of the effectiveness of MBSR for individuals who have difficulties in sleep have been published. Therefore, the aim of this review is to update and synthesize evidence on the effects of MBSR for people with sleep disturbance or insomnia.
\end{abstract}

Methods/design: We will search ovidMEDLINE, ovidEMBASE, Allied and Alternative Medicine, PsycINFO, the Cochrane Central Register of Controlled Trials, the Cumulative Index to Nursing and Allied Health Literature, and four representative Korean electronic databases including KoreaMed, the Korean Studies Information Service System, the Korean Medical Database, and the National Digital Science Library. Two reviewers will independently screen and select relevant studies. The Cochrane Risk of Bias tool will be used to assess risk of bias in the included studies. The primary outcomes will be defined as the change in sleep quality before and after the intervention as evaluated by the polysomnography or self-reported questionnaires such as the Pittsburgh Sleep Quality Index. If quantitative synthesis is not appropriate, a descriptive analysis might be undertaken.

Discussion: Many published primary studies have investigated the positive effects of MBSR on sleep quality; however, there are no systematic reviews and meta-analyses synthesizing the evidence of up-to-date research on the effects of MBSR for sleep problems. The review findings will aid the general population and healthcare providers in making informed decisions on evidence-based intervention selection for sleep disturbance or insomnia. Systematic review registration: PROSPERO CRD42015027963.

Keywords: Mindfulness, Sleep disorders, Systematic review, Protocol

\footnotetext{
* Correspondence: shj5th@korea.ac.kr

Seong Min Kim and Jeong Min Park are co-first authors.

${ }^{\dagger}$ Equal contributors

${ }^{3}$ Department of Nursing, College of Medicine, Chosun University, 309

Pilmum-daero, Dong-gu, Gwangju 61452, South Korea

Full list of author information is available at the end of the article
} 


\section{Background}

Sleep disturbance is recognized as a public health concern. Approximately $12.2 \sim 39.4 \%$ of general population drawn from different countries were affected by symptoms of insomnia, such as difficulty initiating or maintaining sleep, waking up too early, and in some cases, having non-restorative or poor quality of sleep [1-4]. An estimated $50 \%$ of older adults claimed to have difficulty initiating and maintaining sleep [5]. Sleep problems can lead to long-term physical and mental disorder, and linked to psychiatric conditions such as depression [6], anxiety [7], mood disorders [8], and dementia [9]. So, sleep disturbance deteriorate an individual's quality of life and puts a significant economic burden on society [10]. Reduced productivity resulting from chronic insomnia in the US workforce has been estimated to cost $\$ 63.2$ billion annually [11]. An additional $\$ 32$ billion is spent by US consumers each year in the "sleep market" (e.g., hypnotics, sleep masks, and white noise devices) [12]. Therefore, treatment and management for sleep disturbances is a critical part of caring for people with sleep difficulties and insomnia symptoms.

Mindfulness-based stress reduction (MBSR) has been used as a complementary and alternative medicine therapy for alleviating stress and anxiety, and has been shown to play a role in alleviating symptoms of insomnia. MBSR is based on the Buddhist philosophy and was created by Jon Kabat-Zinn [13]. Kabat-Zinn defines mindfulness as "paying attention in a particular way: on purpose, in the present moment, and nonjudgmentally" [13]. This refers to "know awareness mind as" without the intervention of the subjective thoughts and feelings. Improving the mental and physical health through them and promotes insight into the meaning of life and physical changes leading to tranquility and peace of mind [14]. One approach to addressing insomnia that is rooted in addressing cognitive arousal is mindfulnessbased training [15]. This practice allows individuals to focus on the present and let go of the thoughts, beliefs, and emotions that create stress [16]. In addition, it allows individuals to focus on the mental and physical states that lead to a positive response to sleep, and to avoid reacting in a negative way to sleep disturbances [15]. Thus, insomnia might be improved through an MBSR training program [16].

In previous systematic review evaluating the effectiveness of MBSR for people with sleep disturbance, there was some evidence to suggest that MBSR might improve sleep and decrease sleep-interfering cognitive processes [17]. However, due to the lack of standardized outcome measures of studies included in the review, a metaanalysis was not undertaken. And the authors of the review concluded that the well-designed randomized controlled trials (RCTs) are needed to elucidate the effects of MBSR practice. Recently, several RCTs of the effectiveness of MBSR for individuals who have difficulties in sleep have been published [18-21]. Therefore, the aim of this systematic review is to update and evaluate evidence on effectiveness of MBSR for peoples with sleep disturbance or insomnia.

\section{Methods/design}

The systematic review and meta-analysis will be conducted in accordance with the Preferred Reporting Items for Systematic Reviews and Meta-Analyses (PRISMA) guidelines [22]. The following protocol has been reported in accordance with the PRISMA-P guidelines (Additional file 1). The protocol has been registered with the PROSPERO International Prospective Register of Systematic Reviews (CRD42015027963).

\section{Selection criteria \\ Study design \\ RCTs will be included without date and language restriction.}

\section{Population}

All adults (at least 18 years old) regardless of health conditions were included, excepting shift-workers and travelers. It was not required to be diagnosed with sleep disorders.

\section{Intervention}

The MBSR programs will comprise standardized programs lasting for several weeks, including 6- to 10-week courses of 1 - to 2.5 -h sessions, or 1-day intensive training sessions. We will also include daily home practice programs [23]. Programs will need to be conducted by certified mindfulness teachers and delivered to an individual or group either face-to-face or online. The mindfulness practices covered will include mindful sitting meditation, mindful eating, appreciation meditation, friendly or loving-kindness meditation, mindful walking, and mindful movement [24]. We will include all studies utilizing MBSR, allowing for slight deviations from the original practices developed by Kabat-Zinn [13]. However, other mindfulness mediation practices such as mindfulness-based cognitive therapy, Vipassana, acceptance and commitment therapy, or Zen will be excluded from the review. In other words, only MBSR programs were of interest.

\section{Comparators}

The control group can be either passive (i.e., waitlist) and/or active (e.g., receiving other standard care or sleep hygiene education) [18-22]. 


\section{Outcomes}

We will assess the following outcome measures based on analyses of the data obtained in the included trials. Studies lacking standardized outcome measures (e.g., where primary outcomes are demonstrated using only graphs) will be excluded from the quantitative data analysis. The primary outcomes will be defined as the change in sleep quality before and after interventions. Objective sleep quality is measured by polysomnography or wrist actigraphy $[19,21]$. Patient-reported outcome measures were total sleep time, sleep efficiency, sleep onset latency, and wake after sleep onset from sleep diaries, or self-reported questionnaires such as the Pittsburgh Sleep Quality Index and Insomnia Severity Index $[18,20]$. The secondary outcomes considered will be sleep-related daytime impairments (depressive symptoms, anxiety symptoms, perceived stress, and fatigue symptoms) and quality of life [18, 20, 21].

Trials in which MBSR was used in a combination modality (e.g., in combination with different meditation techniques or hypnotics) will be excluded if the treatment effect of MBSR could not be isolated.

\section{Search strategy}

The following databases will be searched for relevant studies from their inception of database until October 2015: ovidMEDLINE, ovidEMBASE, Allied and Alternative Medicine (AMED), PsycINFO, the Cochrane Central Register of Controlled Trials (CENTRAL), and the $\mathrm{Cu}$ mulative Index to Nursing and Allied Health Literature (CINAHL). Manual searches for additional studies will also be performed by reviewing the reference lists of relevant studies. We will also search for studies in four representative Korean databases, including KoreaMed, the Korean Studies Information Service System (KISS), the Korean Medical Database (KMbase), and the National Digital Science Library (NDSL).

Additional file 2 presents the full list of search terms that will be used, and the search terms were adapted for the other databases. Other relevant trials will be searched for by manually screening the reference lists of trials and relevant review papers identified in the initial searches. In addition, the corresponding authors of selected studies will be contacted to supplement incomplete information.

Ongoing clinical trials will be searched for on Clinical trials.gov (https://clinicaltrials.gov/ct2/home) and the World Health Organization International Clinical Trials Registry Platform (WHO ICTRP; http://apps.who.int/trialsearch/).

\section{Study selection}

The reference software program EndNote (EndNote X7, Thomson Reuters, New York, USA) will be used to manage articles and remove duplicate references. Two independent reviewers (KSM and PJM) will select the studies according to the eligibility criteria. Selection process will be as follows: the first, titles and abstracts of the retrieved records will be screened to identify relevant studies. In cases of inconsistencies, the full text will be read. The second, full text of the articles selected in the first stage will be assessed and scrutinized. Disagreements will be discussed and resolved by consensus, if necessary, a third reviewer $(\mathrm{SHJ})$ will be consulted. The details of the selection process will be presented in the Preferred Reporting Items for Systematic review and Meta-Analysis (PRISMA) flow diagram (Fig. 1) [25]. The excluded studies will be listed in a supplementary file along with our reasons for exclusion.

\section{Assessment of risk of bias in included studies}

The reviewers will independently assess the methodological quality of the included studies using the Cochrane Risk of Bias (RoB) tool [26]. The RoB tool comprises seven specific domains addressing five types of bias: random sequence generation (selection bias), allocation concealment (selection bias), blinding of participants and personnel (performance bias), blinding of outcome assessment (detection bias), incomplete outcome data (attrition bias), selective outcome reporting (reporting bias), and other sources of bias. We will judge the risk of bias of each study as "high," "unclear," or "low" according to the results of the RoB tool. Any disagreement will be resolved via consensus or consultation with a third reviewer.

\section{Data extraction}

Before data extraction, we will devise a standardized form containing the specified outcomes via a discussion among all reviewers. Two reviewers will then extract data independently using this standardized form. Any disagreements regarding extracted data will be resolved via consensus with a third person. The data extraction form will include the study design, country of publication, number and characteristics of participants, details of the MBSR program, follow-up periods, and primary/ secondary outcomes. Where reported data are insufficient or ambiguous, reviewers will contact the corresponding authors by e-mail requesting additional clarification or information.

\section{Data synthesis}

Dichotomous data will be reported as the risk ratio (RR) with $95 \%$ confidence intervals (CIs). For continuous data, we will use the mean difference (MD) to measure the treatment effects with $95 \%$ CIs. However, if the outcome variables were measured using different scales, we 


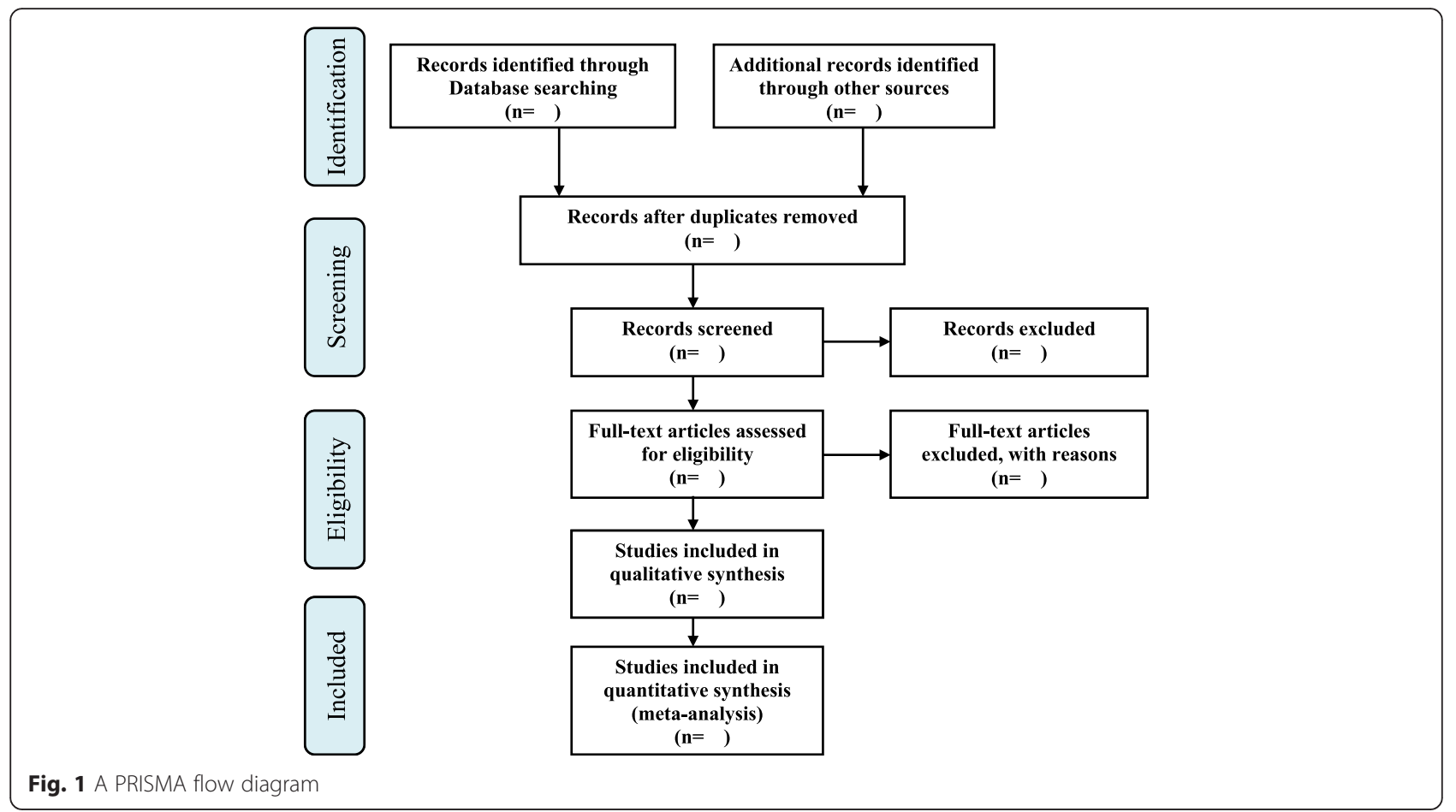

will use the standardized mean difference (SMD) with 95 \% CIs.

A meta-analysis will be carried out where appropriate to synthesize data from similar studies using Review Manager (V.5.3) and STATA 10.0 (Stata Corp., College Station, Texas). Using the DerSimonian method and the Laird random-effects model, the pooled estimates of the weighted mean difference (WMD) or SMD with $95 \%$ CIs will be calculated for continuous outcomes and RRs with $95 \%$ CIs for dichotomous outcomes [27]. We will assess heterogeneity of treatment effects across studies using the $I^{2}$ and the Q-statistic [28]. An $I^{2}$ value of $>50 \%$ will be considered an indication of substantial heterogeneity [29], and if heterogeneity is observed, we will conduct a subgroup analysis. Where possible, we will perform a subgroup analysis (1) by type of sleep disturbance (e.g., the severity of sleep disturbance or insomnia) and (2) by duration of MBSR (e.g., shortterm treatment of 8 weeks or long-term treatment of more than 8 weeks). A sensitivity analysis will be considered for studies that can be grouped based on their methodological quality and funding sources. A Funnel plot will be used to detect any publication bias if more than 10 studies are included in this analysis [28]. Additionally, we will perform Egger's statistical test to detect any possible publication bias (using the criterion of $p<0.1$ ) [30]. If a quantitative synthesis is not appropriate, a narrative synthesis will be conducted to interpret the data.

\section{Quality of evidence and dissemination of findings}

We will use the Grading of Recommendations, Assessment, Development and Evaluation (GRADE) approach to judge the quality of evidence for outcomes of interest. This approach considers the following aspects to assess the quality of a body of evidence: study design, the risk of bias, imprecision of results, inconsistency across studies, application of results to the population of interest, and likelihood of publication bias [31]. Quality will be rated as high, moderate, low, or very low. The results will be disseminated through peer-reviewed publication and international conferences.

\section{Discussion}

MBSR is widely applied in both clinical populations and healthy individuals to alleviate stress and stress-related health conditions such as cardiac diseases [32] and longterm physical conditions [33], as well as to promote health [34, 35]. Sleep disturbances are considered a significant medical and public health concern. However, it remains unclear whether MBSR can be used to alleviate sleep difficulties [17]. As there are several RCTs on the effects of MBSR on sleep problems [36-38], we thought it is necessary to provide an up-to-date systematic review of the evidence on MBSR. Therefore, the results from the review proposed herein will be carefully interpreted according to study quality, the content of the MBSR program, and the clinical importance of the outcome measures used in the included studies. The findings of this review will aid both the general population 
and healthcare providers to make informed decisions on evidence-based intervention alternatives for sleep disturbance or insomnia.

\section{Limitations}

Because our database searches will be limited to published articles, bias might be introduced through the exclusion of unpublished data from thesis and conference proceedings abstracts. This publication bias might inflate the treatment effect estimates because studies with desirable or significant results are more likely to be granted publication. If we suspect any publication bias, Duval's trim and fill method will be used to correct for it [39].

\section{Additional files}

Additional file 1: PRISMA-P checklist. (DOCX $19 \mathrm{~kb}$ )

Additional file 2: Sample search strategy for ovidMEDLINE. (DOC 28 kb)

\section{Abbreviations}

MBSR: mindfulness-based stress reduction; RCT: randomized controlled trial.

\section{Competing interests}

The authors declare that they have no competing interests.

\section{Authors' contributions}

$\mathrm{SHJ}$ conceived the systematic review. The review protocol was drafted by KSM and PJM and revised by SHJ. SHJ and KSM participated in the development of the search strategies. KSM and PJM will independently select the potential studies, assess the risk of bias, and extract the data from the included studies. SHJ will help to resolve any disagreements and ensure that no errors occur during the review. All authors have read and approved the final version of this review protocol.

\section{Acknowledgements}

There is no financial support for this study.

\section{Author details}

'Department of Nursing, College of Medicine, Seonam University, Namwon, South Korea. ${ }^{2}$ Graduate School of Chosun University, Gwangju, South Korea. ${ }^{3}$ Department of Nursing, College of Medicine, Chosun University, 309 Pilmum-daero, Dong-gu, Gwangju 61452, South Korea.

Received: 13 November 2015 Accepted: 21 March 2016 Published online: 02 April 2016

\section{References}

1. Roth T. Insomnia: definition, prevalence, etiology, and consequences. J Clin Sleep Med. 2007;3(5 Suppl):S7-10.

2. Fielding $R$, Wong WS. Prevalence of chronic pain, insomnia, and fatigue in Hong Kong. Hong Kong Med J. 2012;18 Suppl 3:9-12.

3. Benbir G, Demir AU, Aksu M, Ardic S, Firat H, Itil O, Ozgen F, Y.lmaz H, Karadeniz D. Prevalence of insomnia and its clinical correlates in a general population in Turkey. Psychiatry Clin Neurosci. 2015;69(9):543-52. doi:10.1111/pcn.12252.

4. Ohayon MM, Hong SC. Prevalence of insomnia and associated factors in South Korea. J Psychosom Res. 2002;53(1):593-600.

5. Crowley K. Sleep and sleep disorders in older adults. Neuropsychol Rev. 2011:21(1):41-53. doi:10.1007/s11065-010-9154-6.

6. Fernandez-Mendoza J, Shea S, Vgontzas AN, Calhoun SL, Liao D, Bixler EO. Insomnia and incident depression: role of objective sleep duration and natural history. J Sleep Res. 2015;24(4):390-8.

7. Choueiry N, Salamoun T, Jabbour H, El Osta N, Hajj A, Rabbaa Khabbaz L. Insomnia and relationship with anxiety in university students: a crosssectional designed study. PLoS One. 2016:11(2):e0149643. doi:10.1371/ journal.pone.0149643.
8. Rumble ME, White $\mathrm{KH}$, Benca RM. Sleep disturbances in mood disorders. Psychiatr Clin North Am. 2015;38(4):743-59. doi:10.1016/j.psc.2015.07.006.

9. Spira AP, Chen-Edinboro LP, Wu MN, Yaffe K. Impact of sleep on the risk of cognitive decline and dementia. Curr Opin Psychiatry. 2014;27(6):478-83. doi:10.1097/YCO.0000000000000106.

10. Morin CM, LeBlanc M, Belanger L, Ivers H, Mérette C, Savard J. Prevalence of insomnia and its treatment in Canada. Can J Psychiatry. 2011;56:540-8.

11. Kessler RC, Berglund PA, Coulouvrate C, Roth T, Shahly V, Shillington AC, et al. Insomnia and the performance of US workers results from the American Insomnia Survey. Sleep. 2001;34:1161-71.

12. Fottrell Q. The eye-opening costs of America's insomnia. In Wall Street Journal Market Watch. 2012. http://www.marketwatch.com/story/theshocking-price-of-insomnia-in-america-2012-12-20. Accessed 14 July 2015.

13. Kabat-Zinn J. Full catastrophe living: Using the wisdom of your body and mind to face stress, pain, and illness. New York: Delacorte; 1990

14. Kabat-Zinn J. Wherever you go there you are. New York: Hyperion; 1994

15. Ong J, Sholtes D. A mindfulness-based approach to the treatment of insomnia. J Clin Psychol. 2010;66:1175-84.

16. Ong JC, Ulmer CS, Manber R. Improving sleep with mindfulness and acceptance: a metacognitive model of insomnia. Behav Res Ther. 2012; 50(11):651-60. doi:10.1016/j.brat.2012.08.001.

17. Winbush NY, Gross CR, Kreitzer MJ. The effects of mindfulness-based stress reduction on sleep disturbance: a systematic review. Explore (NY). 2007:3:585-91.

18. Andersen SR, Würtzen H, Steding-Jessen M, Christensen J, Andersen KK, Flyger $\mathrm{H}$, Mitchelmore C, Johansen C, Dalton SO. Effect of mindfulnessbased stress reduction on sleep quality: results of a randomized trial among Danish breast cancer patients. Acta Oncol. 2013;52(2):336-44. doi:10.3109/ 0284186X.2012.745948.

19. Lengacher $C A$, Reich RR, Paterson CL, Jim HS, Ramesar S, Alinat CB, Budhrani $\mathrm{PH}$, Farias JR, Shelton MM, Moscoso MS, Park JY, Kip KE. The effects of mindfulness-based stress reduction on objective and subjective sleep parameters in women with breast cancer: a randomized controlled trial. Psychooncology. 2015;24(4):424-32. doi:10.1002/pon.3603.

20. Cash E, Salmon P, Weissbecker I, Rebholz WN, Bayley-Veloso R, Zimmaro LA, Floyd A, Dedert E, Sephton SE. Mindfulness meditation alleviates fibromyalgia symptoms in women: results of a randomized clinical trial. Ann Behav Med. 2015;49(3):319-30. doi:10.1007/s12160-014-9665-0.

21. Gross CR, Kreitzer MJ, Reilly-Spong M, Wall M, Winbush NY, Patterson R, Mahowald M, Cramer-Bornemann M. Mindfulness-based stress reduction versus pharmacotherapy for chronic primary insomnia: a randomized controlled clinical trial. Explore (NY). 2011;7(2):76-87. doi:10.1016/j.explore.2010.12.003.

22. Moher D, Liberati A, Tetzlaff J, Altman DG, PRISMA Group. Preferred reporting items for systematic reviews and meta-analyses: the PRISMA statement. J Clin Epidemiol. 2009;62(10):1006-12. doi:10.1016/j.jclinepi.2009.06.005.

23. Kabat-Zinn J, Hanh T. Full catastrophe living: using the wisdom of your body and mind to face stress, pain, and illness. New York: Random House Publishing Group; 2009.

24. Black DS, O'Reilly GA, Olmstead R, Breen EC, Irwin MR. Mindfulness meditation and improvement in sleep quality and daytime impairment among older adults with sleep disturbances: a randomized clinical trial. JAMA Intern Med. 2015;175(4):494-501. doi:10.1001/jamainternmed.2014.8081.

25. Liberati A, Altman DG, Tetzlaff J, Mulrow C, Gøtzsche PC, loannidis JP, et al. The PRISMA statement for reporting systematic reviews and meta-analyses of studies that evaluate healthcare interventions: explanation and elaboration. BMJ. 2009:339:b2700. doi:10.1136/bmj.b2700.

26. Higgins JPT, Altman DG, Sterne JAC. Chapter 8: assessing risk of bias in included studies. In: Higgins JPT, Green S. eds. Cochrane handbook for systematic reviews of interventions version 5.1.0 (updated March 2011). The Cochrane Collaboration, 2011. http://www.cochrane-handbook.org. Accessed 14 August 2015

27. DerSimonian R, Laird N. Meta-analysis in clinical trials. Control Clin Trials. 1986;7(3):177-88.

28. Sterne JAC, Egger M, Moher D. Chapter 10: addressing reporting biases. In: Higgins JPT, Green S. eds. Cochrane handbook for systematic reviews of interventions version 5.1.0 (updated March 2011). The Cochrane Collaboration, 2011. http://www.cochrane-handbook.org. Accessed 14 August 2015.

29. Schroll JB, Moustgaard R, Gotzsche PC. Dealing with substantial heterogeneity in Cochrane reviews. Cross-sectional study. BMC Med Res Methodol. 2011;11:22. doi:10.1186/1471-2288-11-22.

30. Egger M, Davey Smith G, Schneider M, et al. Bias in meta-analysis detected by a simple, graphical test. BMJ. 1997;315:629-34. 
31. Murad MH, Montori VM, loannidis JP, Jaeschke R, Devereaux PJ, Prasad K, et al. How to read a systematic review and meta-analysis and apply the results to patient care: users' guides to the medical literature. JAMA. 2014; 312(2):171-9. doi:10.1001/jama.2014.5559.

32. Younge JO, Gotink RA, Baena CP, Roos-Hesselink JW, Hunink MM. Mindbody practices for patients with cardiac disease: a systematic review and meta-analysis. Eur J Prev Cardiol. 2014;22(11):1385-98. doi:10.1177/ 2047487314549927.

33. Crowe M, Jordan J, Burrell B, Jones V, Gillon D, Harris S. Mindfulness-based stress reduction for long-term physical conditions: A systematic review. Aust N Z J Psychiatry. 2016;50(1):21-32. doi:10.1177/0004867415607984.

34. Sharma M, Rush SE. Mindfulness-based stress reduction as a stress management intervention for healthy individuals: a systematic review. J Evid Based Complementary Altern Med. 2014;19(4):271-86. doi:10.1177/ 2156587214543143.

35. Khoury B, Sharma M, Rush SE, Fournier C. Mindfulness-based stress reduction for healthy individuals: a meta-analysis. J Psychosom Res. 2015; 78(6):519-28. doi:10.1016/j.jpsychores.2015.03.009.

36. Cincotta AL, Gehrman P, Gooneratne NS, Baime MJ. The effects of a mindfulness-based stress reduction programme on pre-sleep cognitive arousal and insomnia symptoms: a pilot study. Stress and Health. 2011;27(3):e299-305. doi:10.1002/smi.1370

37. Zhang JX, Liu XH, Xie XH, Zhao D, Shan MS, Zhang XL, et al. Mindfulness-based stress reduction for chronic insomnia in adults older than 75 years: a randomized, controlled, single-blind clinical trial. Explore (NY). 2015;11(3):180-5. doi:10.1016/j.explore.2015.02.005

38. Ong JC, Manber R, Segal Z, Xia Y, Shapiro S, Wyatt JK. A randomized controlled trial of mindfulness meditation for chronic insomnia. Sleep. 2014; 37(9):1553-63. doi:10.5665/sleep.4010.

39. Duval S, Tweedie R. Trim and fill: a simple funnel-plot-based method of testing and adjusting for publication bias in meta-analysis. Biometrics. 2000; 56:455-63.

\section{Submit your next manuscript to BioMed Central and we will help you at every step:}

- We accept pre-submission inquiries

- Our selector tool helps you to find the most relevant journal

- We provide round the clock customer support

- Convenient online submission

- Thorough peer review

- Inclusion in PubMed and all major indexing services

- Maximum visibility for your research

Submit your manuscript at www.biomedcentral.com/submit 